ARTICLE

https://doi.org/10.1038/s41467-019-11989-x

\title{
The CONSTANS flowering complex controls the protective response of photosynthesis in the green alga Chlamydomonas
}

\author{
Ryutaro Tokutsu (1) 1,2, Konomi Fujimura-Kamada1, Takuya Matsuo ${ }^{3}$, Tomohito Yamasaki (i) ${ }^{4}$ \& \\ Jun Minagawa (10) ${ }^{1,2}$
}

Light is essential for photosynthesis, but the amounts of light that exceed an organism's assimilation capacity can result in oxidative stress and even cell death. Plants and microalgae have developed a photoprotective response mechanism, $\mathrm{qE}$, that dissipates excess light energy as thermal energy. In the green alga Chlamydomonas reinhardtii, $\mathrm{qE}$ is regulated by light-inducible photoprotective proteins, but the pathway from light perception to $\mathrm{qE}$ is not fully understood. Here, we show that the transcription factors CONSTANS and Nuclear transcription Factor Ys (NF-Ys) form a complex that governs light-dependent photoprotective responses in $C$. reinhardtii. The qE responses do not occur in CONSTANS or NF-Y mutants. The signal from light perception to the CONSTANS/NF-Ys complex is directly inhibited by the SPA1/COP1-dependent E3 ubiquitin ligase. This negative regulation mediated by the E3 ubiquitin ligase and the CONSTANS/NF-Ys complex is common to photoprotective response in algal photosynthesis and flowering in plants.

\footnotetext{
${ }^{1}$ Division of Environmental Photobiology, National Institute for Basic Biology, Nishigo-naka 38, Myodaiji, Okazaki 444-8585, Japan. ${ }^{2}$ Department of Basic Biology, School of Life Science, Graduate University for Advanced Studies, Okazaki 444-8585, Japan. ${ }^{3}$ Center for Gene Research, Nagoya University, Nagoya 464-8602, Japan. ${ }^{4}$ Science and Technology Department, Natural Science Cluster, Kochi University, 2-5-1 Akebono-cho, Kochi 780-8520, Japan. Correspondence and requests for materials should be addressed to R.T. (email: tokutsu@nibb.ac.jp) or to J.M. (email: minagawa@nibb.ac.jp)
} 
$\mathrm{P}$ hotosynthetic organisms are often exposed to excess light. This can result in severe oxidative stress and even cell death. Plants and algae have developed a photoprotective response mechanism, $\mathrm{qE}$, which is driven by low $\mathrm{pH}$ in the thylakoid lumen generated by the photosynthetic electron flow ${ }^{1}$. This lumenal low $\mathrm{pH}$ modifies the light-harvesting complex II pigment composition via the xanthophyll cycle and activates specific photoprotective proteins, such as PSBS and LIGHTHARVESTING COMPLEX STRESS-RELATED PROTEINS $(\text { LHCSRs })^{2}$. Mutants deficient in these effector proteins are highly stressed under high light (HL) conditions ${ }^{3,4}$. In a green alga Chlamydomonas reinhardtii, qE-type photoprotection is lightinducible via the expression of photoprotective proteins (LHCSRs and PSBS $)^{5}$. Two photoreceptors-PHOTOTROPIN (PHOT) ${ }^{6}$ and ultra-violet (UV) RESISTANCE LOCUS 8 (UVR8) ${ }^{7}$-were identified as initiation factors for signal transduction of the photoprotective genes and protein expression in the green alga. Subsequently, two phot suppressor loci were identified to be involved in the induction of LHCSRs: DE-ETIOLATED 1 (det1) and DAMAGED DNA-BINDING $1(d d b 1)$ and an E3 ubiquitin ligase complex, CUL4-DDB1 ${ }^{\mathrm{DET} 1}$, was proposed to mediate the PHOT signal to LHCSRs gene expression ${ }^{8}$. Despite these findings, we still do not know most of other important players in the signal transduction pathways, especially transcription factors that directly activate light-dependent gene expression. During a recent genetic screening using a bioluminescence reporter assay, however, we obtained several Deficient in LHCSR expression (DSR) mutants that showed reduced expression of LHCSR genes ${ }^{9}$. Two of the mutants, DSR10 and DSR15, had a mutation in CONSTANS and two other mutants, DSR28 and CC4286, had a mutation in $N F-Y B$.

CONSTANS (CO) is a circadian clock-regulated gene encoding a transcription factor required for flowering. Since Coupland et al. first reported that $\mathrm{CO}$ mediates photoperiodic flowering by directly regulating the transcription of FLOWERING LOCUS T $(F T)^{10}$, extensive studies have revealed that $\mathrm{CO}$ and its ubiquitination under the control of photoreceptors are the central to regulation of photoperiodic flowering ${ }^{11}$. NUCLEAR FACTOR Y (NF-Y) proteins (NF-YA, NF-YB, and NF-YC) bind to the promoter regions of their target genes ${ }^{12}$ and are widely conserved in eukaryotic organisms, including yeast, mammals, and plants. NFYs are the most thoroughly studied proteins that interact with $\mathrm{CO}^{13}$. The biological functions and underlying molecular mechanisms of NF-Y proteins have been intensively investigated in both animals and plants ${ }^{14}$. For example, Wenkel et al. reported that in flowering plants, HEME ACTIVATOR PROTEIN, which corresponds to NF-YA, strongly interacts with CO, forming a transcriptional complex that regulates the expression of its downstream gene, $F T^{15}$. The interaction of NF-YB2/3 and NFYC3/4/9 with $\mathrm{CO}$ has physiological relevance in plants, as the absence of any of these proteins results in delayed flowering ${ }^{13}$. A comparable sophisticated mechanism has not been identified in microalgae, as their population growth relies on cell division, not on flowering as in land plants.

Even though C. reinhardtii does not produce flowers, a copy of each of these transcriptional factors has been identified on its genome, including CONSTANS (CrCO), NF-YB, and $N F-Y C^{16,17}$. Previously, heterologous expression of $\mathrm{CrCO}$ was shown to complement the function of $\mathrm{CO}$ in the flowering plant Arabidopsis thaliana ${ }^{16}$. Phylogenetic analysis of $\mathrm{CO}$ and $\mathrm{CO}$-like (COL) genes from green algae and land plants indicated that $\mathrm{CrCO}$ is a precursor of $C O$ in green photosynthetic eukaryotes ${ }^{18}$. The $C$. reinhardtii homologs of NF-YB and NF-YC were clustered with NF-YB1/8/10 and with NF-YC1/2/3/4/9, respectively, in phylogenetic analyses of the NF-Y protein family (Supplementary Fig. $1)^{17}$. Although few physiological and biochemical analyses of algal NF-Ys have been reported to date ${ }^{17}$, these findings suggest that the $\mathrm{CO} / \mathrm{NF}-\mathrm{YB} / \mathrm{NF}-\mathrm{YC}$ transcriptional complex might have arisen before the divergence of land plants. In the current study, using the DSR mutants defective in CrCO or NF-YB and other newly constructed mutants in $C$. reinhardtii, we show that the transcription factors $\mathrm{CrCO}$ and NF-Ys form a complex that governs light-dependent photoprotective responses in $C$. reinhardtii. Further, we show that the CrCO/NF-Ys complex was directly inhibited by the SUPPRESSOR OF PHYA-105 1 (SPA1) and CONSTITUTIVE PHOTOMORPHOGENIC 1 (COP1) -dependent E3 ubiquitin ligase. We propose that $\mathrm{CrCO}$ and its ubiquitination is the central to regulation of photoprotection in $C$. reinhardtii, much as like flowering in plants.

\section{Results}

CONSTANS and NF-YB are crucial for photoprotection. We identified mutations in CrCO in two DSR mutants, DSR10 (crco-1) and DSR15 (crco-2) (Supplementary Fig. 2a Cre06.g278159). A combination of linkage mapping and whole-genome sequencing revealed that two other mutants, DSR28 (nfyb-1) and CC4286 (nfyb-2), had mutations in NF-YB (Supplementary Fig. 2a, Cre02.g079200). While DSR10 (crco-1) and DSR15 (crco-2) had reduced $C r C O$ yet normal NF-YB transcript levels, DSR28 (nfyb-1) and CC4286 ( $n f y b-2)$ had normal CrCO yet reduced $N F-Y B$ transcript levels (Supplementary Fig. 2b). Similarly, RT-PCR analysis revealed that the transcripts of representative genes involved in photoprotection in C. reinhardtii (LHCSRs and PSBSs) were strongly reduced or almost undetectable in all of these mutants $^{9}$ (Supplementary Fig. 3). Under $30 \mu \mathrm{mol}$ photons $\mathrm{m}^{-2} \mathrm{~s}^{-1}$ (low light [LL]) and $100 \mu \mathrm{mol}$ photons $\mathrm{m}^{-2} \mathrm{~s}^{-1}$ (medium light), these mutants had comparable growth rates and similar cell shapes to the wild-type strains (Supplementary Fig. 4). We cannot exclude the possibility that the photoperiodic responses of these mutants were altered as described in the previous report ${ }^{16}$. However, the mutation in $\mathrm{CrCO}$ or $\mathrm{NF}-\mathrm{YB}$ had little effect on the cell division process under low to medium light conditions. These findings suggest that either $\mathrm{CrCO}$ or $\mathrm{NF}-\mathrm{YB}$, or both, are crucial regulators of photoprotective responses in $C$. reinhardtii, and are therefore necessary for survival under HL conditions. This is an intriguing hypothesis, given that their homologs in land plants function together as a flowering module ${ }^{11,13}$.

To test this hypothesis, we first measured photoprotective thermal dissipation of excess absorbed energy in the mutants. This process occurs via the quenching mechanism $\mathrm{qE}$, which is the fastest reversible photoprotective response. It suppresses photo-oxidative damage and contributes to optimizing photosynthetic efficiency under variable light conditions in nature ${ }^{19}$. In C. reinhardtii, $\mathrm{qE}$ type photoprotection is a light-inducible process that involves the expression of photoprotective proteins ${ }^{6,7}$. When DSR15 ( $\left.\mathrm{crco}-2\right)$ and DSR28 (nfyb-1) strains were exposed to excess light (including weak UV), they exhibited pigment bleaching (Fig. 1a, b). This is an indicator of severe photoinhibition and is accompanied by a decrease in the maximum quantum efficiency of photosystem II $(\mathrm{Fv} / \mathrm{Fm})$ (Fig. 1c). These mutations were successfully rescued via expression of either $\mathrm{CrCO}(\mathrm{crco}-2 / \mathrm{CrCO})$ or NF-YB $(n f y b-1 / \mathrm{NFYB})$ fused with a YFP variant, Venus-3xFLAG (Fig. 1a-d). The photoinhibitory phenotype observed in $c r c o-2$ and $n f y b-1$ may have been due to insufficient expression of the key photoprotective proteins, including LHCSR1, LHCSR3, and PSBS, because the mutants' qE activities were almost lost (Fig. 1d, e). These results indicate that both $\mathrm{CrCO}$ and $\mathrm{NF}-\mathrm{YB}$ are essential for the functional activation of $\mathrm{qE}$-dependent photoprotection in C. reinhardtii.

The kinetics of the photoprotective response of the rescued strains were then examined. The $\mathrm{crco}-2 / \mathrm{CrCO}$ strain showed a delayed response in both $\mathrm{qE}$ and photoprotective protein 
a

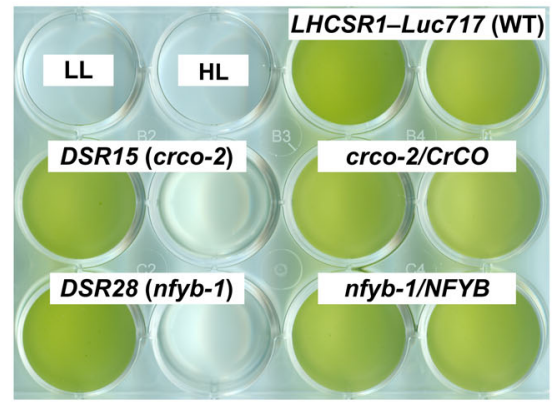

C

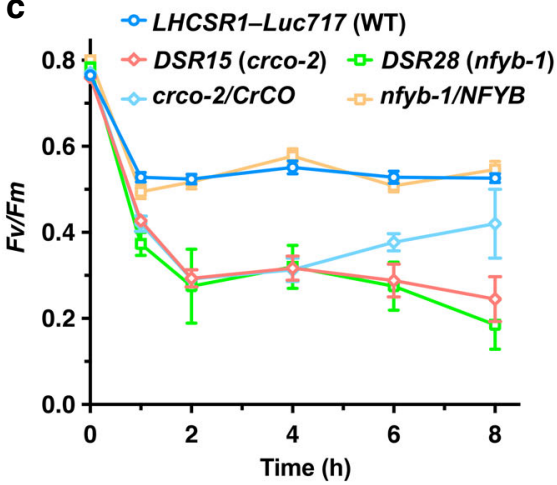

b

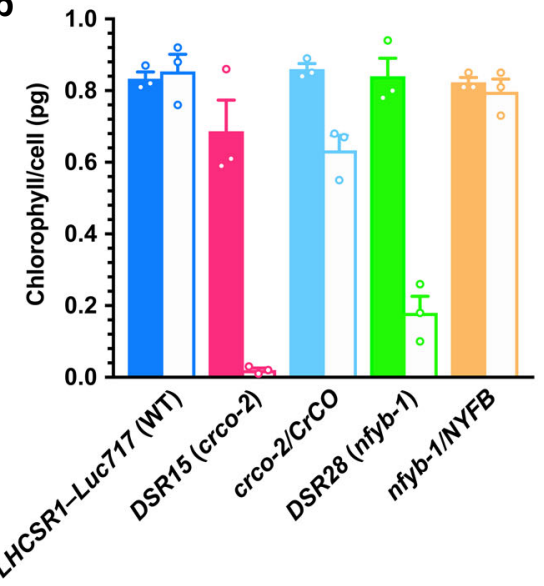

d

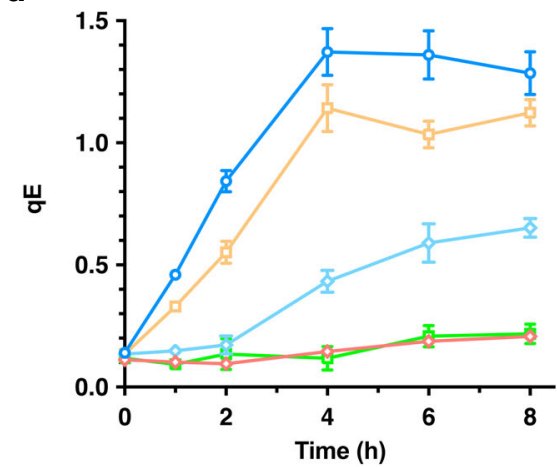

e
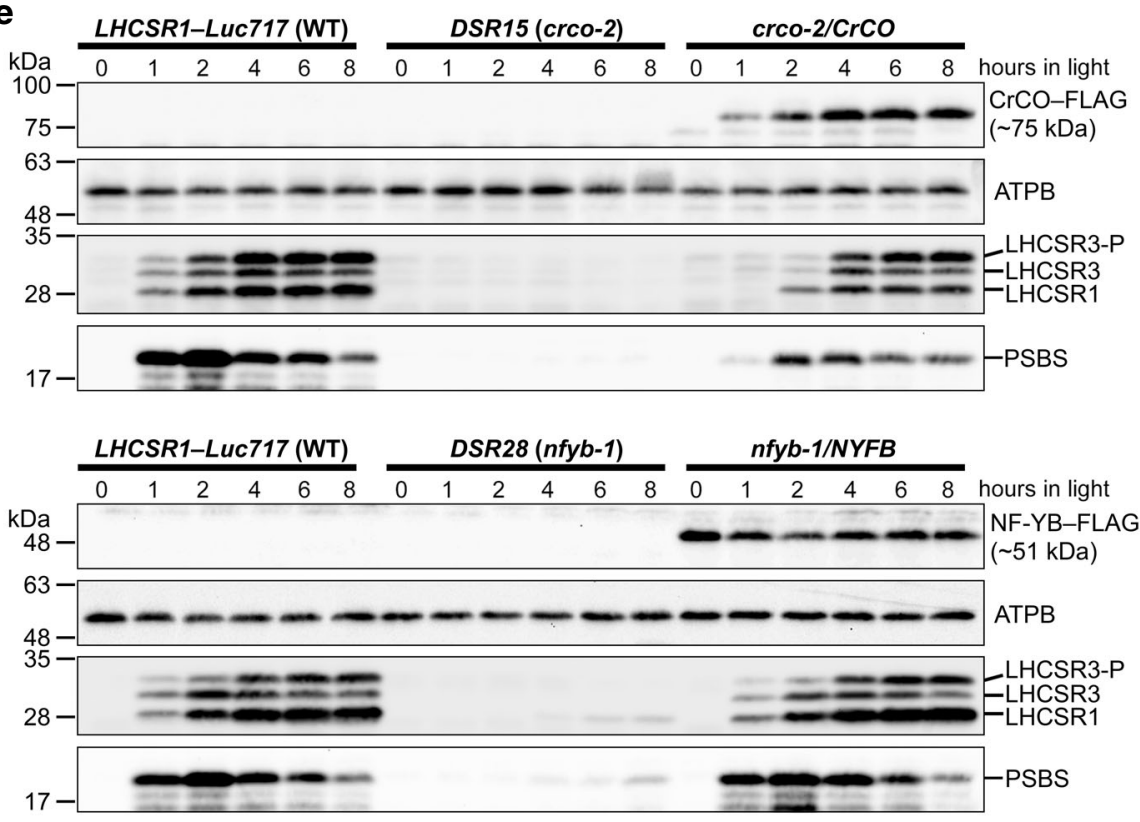

Fig. $1 \mathrm{CrCO}$ and NF-YB are crucial for photoprotection. a The bleaching phenotypes of the reference strain (LHCSRT-LuC717) and the DSR mutants visualized in multiwell plates. Representative cell cultures treated with low light ( $\mathrm{LL}$; left wells) or high light ( $\mathrm{HL}$; right wells). Concentrations of the cultures were adjusted to $1.0 \times 10^{7}$ cells $/ \mathrm{mL}$. b Chlorophyll content per cell after LL (closed bar) or HL (open bar) treatment of the cells shown in a. c Maximum quantum yield of photosystem II (Fv/Fm) during $\mathrm{HL}$ treatment. d qE quenching capability during $\mathrm{HL}$ treatment. e Immunoblot analysis of 3xFLAG-fused proteins (CrCO-FLAG and NF-YB-FLAG in crco-2/CrCO and nfyb-1/NFYB, respectively), LHCSR1, LHCSR3, and PSBS during HL treatment. ATPB protein levels are shown as the loading control. The experiments were performed three times with different biological samples $(n=3$ biological replicates; mean \pm S.E.M); a representative experiment is shown in $\mathbf{e}$ 

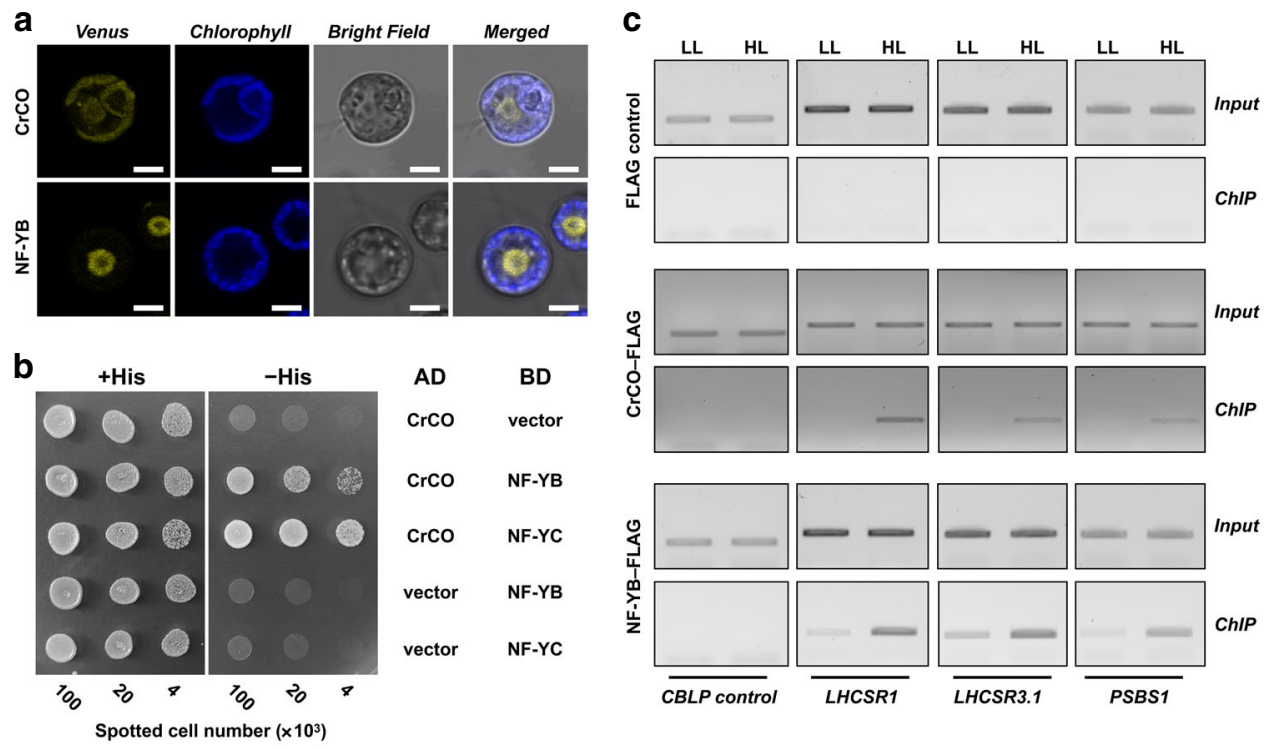

Fig. $2 \mathrm{CrCO}$ interacts with NF-Y isoforms to form a transcriptional complex that associates with the promoter regions of the photoprotective genes. a Confocal live-cell imaging of the complemented strains, crco-2/CrCO (labelled as $\mathrm{CrCO}$ ) and nfyb-1/NFYB (labelled as NF-YB), to visualize the localization of $\mathrm{CrCO}$ and NF-YB fused with Venus-3xFLAG in C. reinhardtii cells. Scale bars, $5 \mu \mathrm{m}$. b GAL4-based yeast two-hybrid (Y2H) assays. CrCO was fused to the GAL4-activation domain (AD-CrCO), whose ability to form heterodimers was tested by cotransformation of the GAL4-binding domain fused with NF-YB or NF-YC (BD-NF-YB or BD-NF-YC). Autoactivation was tested using empty vectors (AD or BD). The cells were plated on permissive ( + His) or selective (-His) media and grown at $30{ }^{\circ} \mathrm{C}$ for $40 \mathrm{~h}$ after being spotted on the plates. c Chromatin immunoprecipitation (ChIP)-PCR assay of $\mathrm{CrCO}$ and NF-YB. Agarose gel electrophoresis showing the strength of the association of $\mathrm{CrCO}$ or NF-YB with the promoter regions of the photoprotective genes under different light treatments. ChIP was performed using $2 \times 10^{8}$ cells $/ \mathrm{mL}$ of $\mathrm{crco}-2 / \mathrm{CrCO}$ (labelled as $\mathrm{CrCO}$ ) or nfyb-1/NFYB (labelled as NF-YB) cells cross-linked with $0.35 \%(\mathrm{v} / \mathrm{v})$ formaldehyde after a 1-h light treatment

expression, whereas the $n f y b-1 / N F Y B$ strain showed a normal photoprotective response (Fig. 1d, e). Considering that the expression kinetics of the key photoprotective proteins (LHCSRs and PSBS) are similar to $\mathrm{CrCO}$ protein accumulation (Fig. 1e), it is likely that the $\mathrm{CrCO}$ protein accumulation or localization in the crco-2/CrCO strain is altered. These results suggest that $\mathrm{CrCO}$ protein accumulation induced by light illumination was responsible for the photoprotective responses in C. reinhardtii.

CONSTANS/NF-Y complex controls photoprotective transcription. During the complementation analysis of $\mathrm{crco}^{2}$ and $n f y b-1$, a Venus-3xFLAG tag was fused to the $\mathrm{C}$ termini of $\mathrm{CrCO}$ and NF-YB, which enabled intracellular live imaging of these proteins. Venus fluorescence was detected near the nucleus in the crco-2/CrCO and $n f y b-1 / N F Y B$ strains. Immunocytochemistry analysis also showed that the DAPI and FLAG signals colocalized in the complemented strains (Supplementary Fig. 5), indicating that $\mathrm{CrCO}$ and NF-YB colocalized in the nucleus (Fig. 2a). Because complex formation among CO, NF-YB, and NF-YC has been reported to be essential for $F T$ regulation in flowering plants ${ }^{20}$, we further tested the possibility of the involvement of NF-YC in the complex by generating and characterizing the NFYC (Cre12.g556400) mutants in C. reinhardtii. The CRISPR-Cas9mediated mutation in NF-YC severely affected both the transcription and translation of the photoprotective factors, in a similar manner to the $\mathrm{CrCO}$ or NF-YB mutants. This resulted in cell death under HL (Supplementary Fig. 6). We subsequently tested the physical interactions between $\mathrm{CrCO}$ and NF-YB, $\mathrm{CrCO}$ and NF-YC, and NF-YB and NF-YC using yeast two-hybrid $(\mathrm{Y} 2 \mathrm{H})$ assays, suggesting that $\mathrm{CrCO}, \mathrm{NF}-\mathrm{YB}$, and NF-YC interacted with each other (Fig. 2b and Supplementary Fig. 7). Similar tripartite interactions among the corresponding proteins have been reported in flowering plants ${ }^{15,20,21}$. These interactions were confirmed using immuno-coprecipitation assays of
CrCO-Venus-3xFLAG and NF-YB-Venus-3xFLAG with FLAG monoclonal antibody followed by LC-MS/MS spectroscopy. This revealed the presence of NF-YB and NF-YC in the precipitates of CrCO-Venus-3xFLAG and NF-YB-Venus-3xFLAG, respectively (Supplementary Tables 1 and 2). Moreover, $\mathrm{CrCO}$ was detected in the precipitate of NF-YB-Venus-3xFLAG (Supplementary Fig. 8). Together, these results strongly indicate that the CO/NF-YB/ NF-YC complex is conserved in C. reinhardtii.

Mutations in $\mathrm{CrCO}$ or NF-YB/NF-YC severely suppressed the transcription of the photoprotective genes in C. reinhardtii (Fig. 1 and Supplementary Fig. 6). Meanwhile, mutations in $C O$ and/or $N F-Y A / N F-Y B / N F-Y C$ in flowering plants have been shown to reduce $F T$ transcription ${ }^{10,15,20}$. All the photoprotective genes including LHCSR1, LHCSR3.1/3.2, and PSBS1/2 have at least one CO-responsive element (CORE), CCACA ${ }^{22}$, and NF-Y ciselement, $C C A A T^{12}$, in the upstream regions of their start codons (Supplementary Fig. 9). It is therefore plausible that $\mathrm{CrCO}$ and NF-YB act as transcription factors that regulate the expression of the photoprotective genes in $C$. reinhardtii.

To investigate whether $\mathrm{CrCO}$ and NF-YB associate with the promoter regions of the photoprotective genes (LHCSR1, LHCSR3.1, and PSBS1) in C. reinhardtii, we performed chromatin immunoprecipitation (ChIP) assays using the cells before and after HL treatment. Both $\mathrm{CrCO}$ and NF-YB associated with the promoter regions of these genes after HL treatment (Fig. 2c), which was quantitated using ChIP-qPCR analysis (Supplementary Fig. 10). NF-YB associated with the promoters of the photoprotective genes in LL, and the association seemed to be facilitated under HL (Supplementary Fig. 10). It has been suggested that the NF-YB/NF-YC complex in A. thaliana binds near CORE elements (CCAAT for NF-YA ${ }^{12}$ and CCACA for $\mathrm{CO}^{22}$ ) to support the association of either NF$\mathrm{YA}$ or $\mathrm{CO}$ with the promoter region ${ }^{23}$. Considering this binding feature of plant NF-YB/NF-YC, it is conceivable that NF-YB 
weakly associates with the promoter region of the photoprotective genes under LL and that this association is reinforced by $\mathrm{CrCO}$ accumulation and the formation of a $\mathrm{CrCO} / \mathrm{NF}-\mathrm{YB} / \mathrm{NF}-$ YC complex under HL. Together with the results of our physiological analysis (Fig. 1), this demonstrates that the $\mathrm{CrCO} / \mathrm{NF}-\mathrm{YB} / \mathrm{NF}-\mathrm{YC}$ transcriptional module is involved in photoprotective processes in $C$. reinhardtii.

UVR8 inhibits E3 ubiquitin ligase to accumulate CONSTANS. In land plants, $\mathrm{CO}$ protein accumulation is controlled by lightdependent post-transcriptional regulation ${ }^{24}$. This regulation is modulated by two CULLIN 4 (CUL4)-based E3 ubiquitin ligase components $^{25}$, SPA1 and COP1, which are involved in the ubiquitination-dependent degradation of $\mathrm{CO}^{26,27}$. The E3 ubiquitin ligase activity of this complex is deactivated by the formation of a protein-protein complex with photoactivated CRYPTOCHROME 2 (CRY2) ${ }^{28}$. The interaction between COP1 and the UV-photoreceptor UVR8 has also reported to be important for UV responses in both land plants ${ }^{29}$ and C. reinhardtii ${ }^{7}$. Using a UVR8-Venus-3xFLAG expression strain, UVinducible nuclear translocation of UVR8 was observed (Fig. 3a and Supplementary Figs. 5 and 11). Additionally, interactions among UVR8, SPA1, and COP1 were identified using a coimmunoprecipitation assay (Fig. $3 \mathrm{~b}$ and Supplementary Tables 3 and 4). These results imply that upon UV perception, UVR8 deactivates E3 ubiquitin ligase through the formation of the UVR8/SPA1/COP1 protein complex as proposed in land plants ${ }^{30}$.

As SPA1 and COP1 are involved in the ubiquitinationdependent proteasomal degradation of $\mathrm{CO}$ in land plants ${ }^{26,27}$, we further investigated the possible interaction among SPA1, COP1, and CrCO. Both SPA1 and COP1 interacted with $\mathrm{CrCO}$ in $\mathrm{Y} 2 \mathrm{H}$ assays (Fig. 3c). Additionally, UV-dependent accumulation of CrCO was observed in the $c r c o-2 / \mathrm{CrCO}$ complemented strain, while it overexpressed $\mathrm{CrCO}$ mRNA even before UV illumination (Fig. 3d and Supplementary Fig. 12). It is therefore likely that $\mathrm{CrCO}$ is degraded in LL by proteasomes after ubiquitination by the SPA1/COP1-dependent E3 ubiquitin ligase complex. This degradation is then inhibited upon exposure to UV.

To further clarify whether the E3 ubiquitin ligase is involved in the accumulation of $\mathrm{CrCO}$ and photoprotective proteins, an spal mutant was analyzed. This mutant has an insertional mutation in the SPA1 gene, and was obtained from the Chlamydomonas mutant library project $(C L i P)$ (see Methods for detail). This mutation mimics deactivation of the SPA1/COP1-dependent E3 ubiquitin ligase. As expected, overexpression of the photoprotective proteins (LHCSRs and PSBS) were observed even under LL conditions in this mutant owing to the accumulation of $\mathrm{CrCO}$ (Fig. 3d). Furthermore, an inhibition of proteasome activity by MG132 treatment of the crco-2/CrCO strain, which resulted in the accumulation of $\mathrm{CrCO}$ protein even under LL conditions (Supplementary Fig. 13), also led to the accumulation of several ubiquitin polypeptides (Cre09. g396400) identified by LC-MS/MS analysis of the coimmunoprecipitated samples of CrCO-Venus-3xFLAG (Supplementary Table. 5). This indicates that $\mathrm{CrCO}$ is actively degraded by proteasomes that are guided by the ubiquitination of the protein. These data further support that SPA1/COP1 modules operate in distinct physiological functions, flowering time control in land plants and photoprotection in $C$. reinhardtii.

The CO/NF-YB/NF-YC complex plays a pivotal role in the photoperiodic control of flowering in land plants through transcriptional regulation of FT expression. Our findings
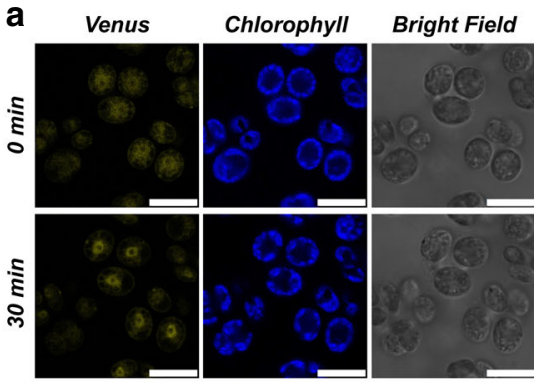

C

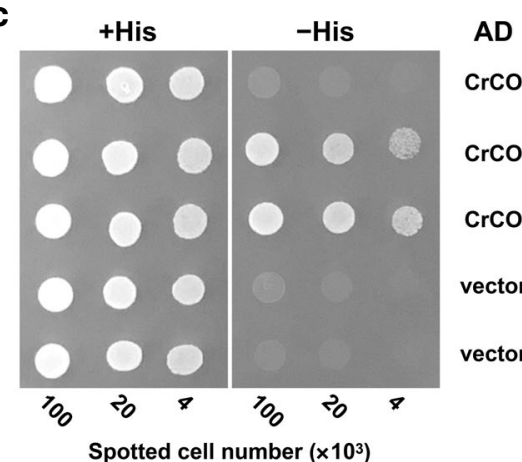

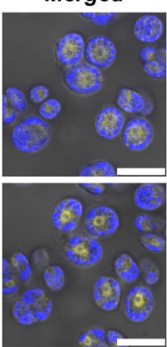

BD

b

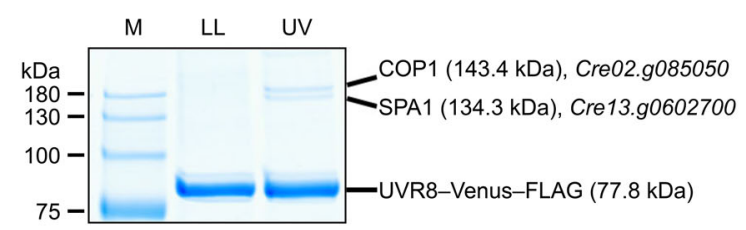

d

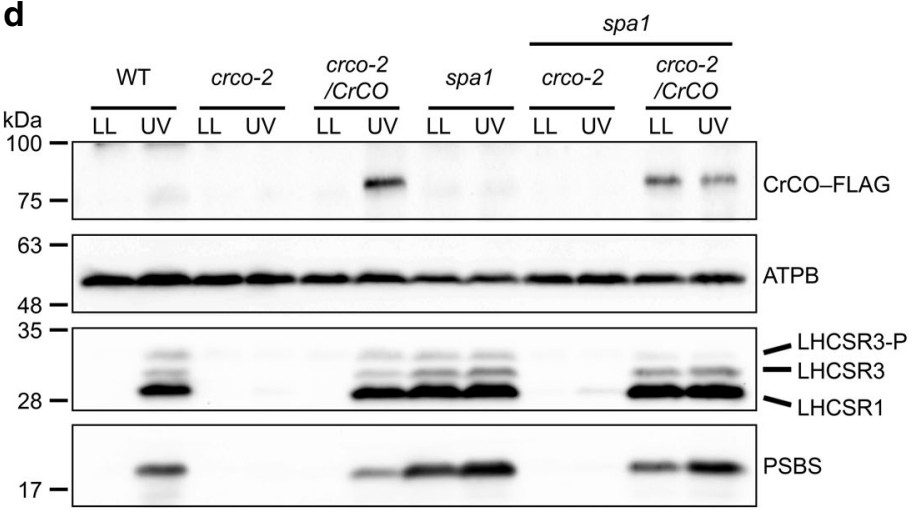

Fig. 3 UVR8 interacts with COP1 and SPA1 to activate the CrCO-based transcriptional complex under UV irradiation. a Confocal images of UVR8-Venus-3xFLAG proteins in the DSR1-comp15 (uvr8/UVR8) strain. The cells were treated with UV for 30 min. Scale bars, $10 \mu \mathrm{m}$. b After $1 \mathrm{~h}$ of UV treatment, the cells were harvested and UVR8-Venus-3xFLAG proteins were immunoprecipitated by FLAG (M2) antibody with SURE-beads. The coimmunoprecipitated proteins were identified by LC-MS/MS analysis of the Coomassie Brilliant Blue-stained polypeptide bands obtained via SDS-PAGE separation after in-gel trypsin digestion. $\mathrm{M}$, molecular mass standard. c Interaction profiles among COP1, SPA1, and CrCO visualized with $\mathrm{Y} 2 \mathrm{H}$ assays, which were performed using COP1, SPA1, and CrCO fused with the AD and/or BD domains of GAL4. The culture conditions were as described for Fig. $2 \mathrm{~b}$. d Immunoblot analysis of LHCSRs, PSBS, and 3xFLAG-fused CrCO to visualize protein accumulation in the wild-type control (WT), crco-2, crco-2/CrCO, spa1, spa1 crco-2, and spa1 crco-2/CrCO strains before and after UV treatment. ATPB proteins were included as the loading controls 


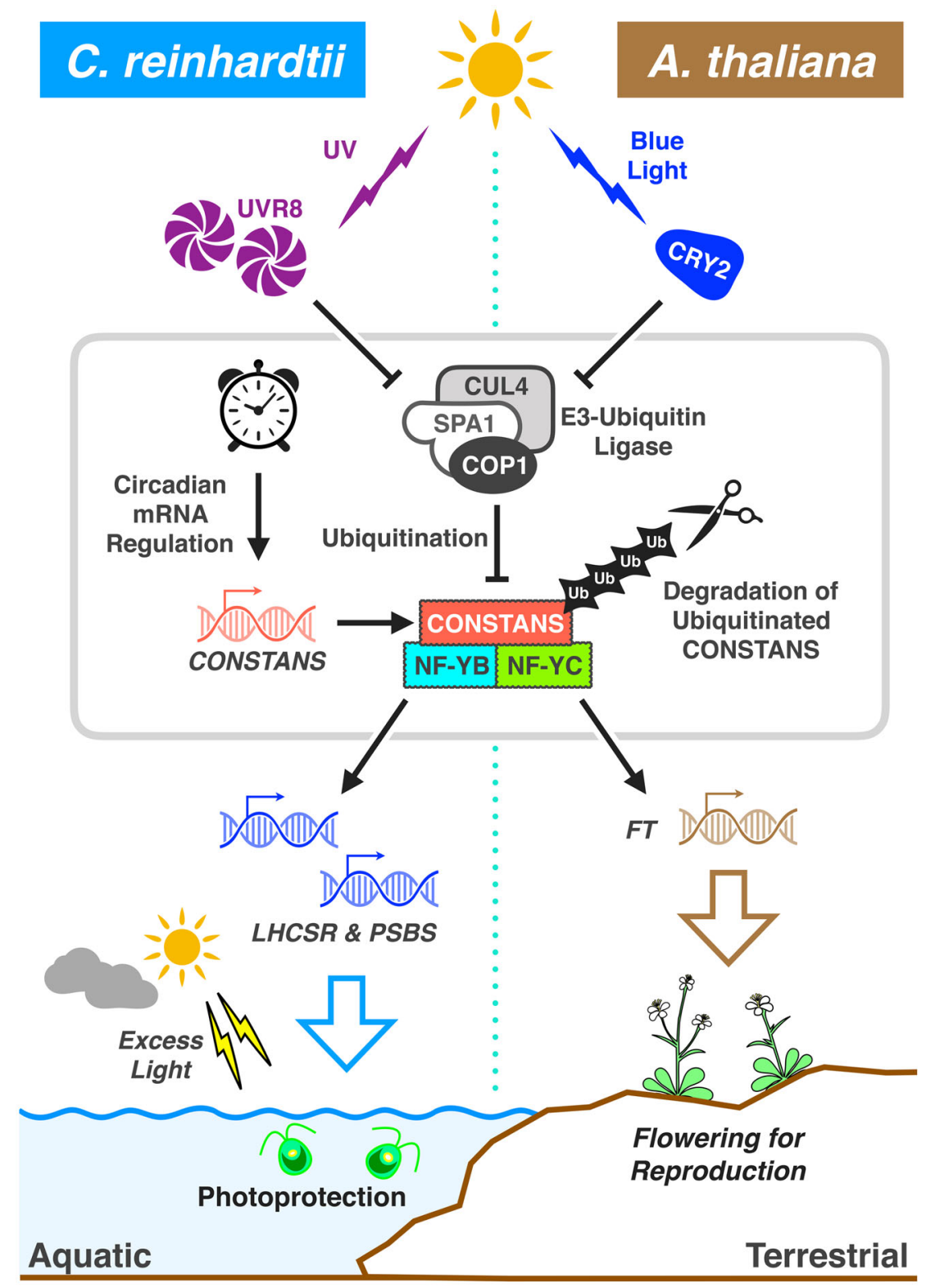

Fig. 4 Hypothetical signal transduction pathways and physiological functions of CONSTANS/NF-YB/NF-YC-dependent control of the target genes in C. reinhardtii and $A$. thaliana. Blue light perception by the photoreceptor CRY2 in A. thaliana deactivates the E3 ubiquitin ligase module ${ }^{27,28}$, whereas UV light perception via conversion of the dimeric form of UVR8 to the monomeric form deactivates this module in C. reinhardtii ${ }^{7}$. Circadian clock-regulated CONSTANS transcripts ${ }^{10}$ are translated, and successfully accumulate in the organisms when proteasome- and ubiquitin-dependent degradation is inactivated. The CONSTANS/NF-YB/NF-YC transcriptional module then transcribes $F T^{10}$ and photoprotective genes (e.g., LHCSR1, LHCSR3, and PSBS) to stimulate flowering and photoprotection in land plants and the green alga, respectively. The kernel, including SPA1/COP1-dependent E3 ubiquitin ligase, circadian regulation of CONSTANS, and the CONSTANS/NF-YB/NF-YC transcriptional complex, was possibly established in an ancestral green photosynthetic organism

therefore suggest that there may also be a link between photoperiodic signaling and the photoprotective response in $C$. reinhardtii. The transcript levels of $\mathrm{CrCO}$ indeed increased during subjective daytime but decreased in subjective night time (Supplementary Fig. 14). This is in part compatible with the previous report that $\mathrm{CrCO}$ transcription is under the control of the circadian clock $^{16}$. Previous studies have also revealed that LHCSR3, formerly known as LI818, displays diurnal oscillations in RNA accumulation under light/dark cycles $^{31,32}$. As $\mathrm{CrCO}$ is critical for survival under HL (Fig. 1), CrCO in fact connects the circadian clock with photoprotective responses in C. reinhardtii (Fig. 4).

\section{Discussion}

Aihara et al. recently reported that another E3 ubiquitin ligase complex, CUL4-DDB1 ${ }^{\mathrm{DET} 1}$, to be involved in the negative regulation of LHCSRs in C. reinhardtii ${ }^{8}$. Since a similar complex has been proposed to enhance the activity of the SPA1/COP1dependent E3 ubiquitin ligase in land plants ${ }^{25}$, the algal CUL4-DDB1 ${ }^{\text {DET1 }}$ may also enhance the ubiquitination at the SPA1/COP1-dependent E3 ubiquitin ligase, thereby transducing the blue light signal to LHCSRs by way of CrCO. Taken together with the synchronized accumulation kinetics of $\mathrm{CrCO}$ and the photoprotective proteins in the $\mathrm{crco}-2 / \mathrm{CrCO}$ strain (Fig. 1e), we propose that the post-translational regulation of $\mathrm{CrCO}$ mediated 
by the E3 ubiquitin ligase, is fundamental for the photoprotective response in C. reinhardtii (Fig. 4).

The initial light input signals differ between flowering plants (in which blue light is perceived by $\mathrm{CRY} 2^{28}$ ) and C. reinhardtii (in which UV is perceived by UVR8). Despite this, the kernel of the downstream regulatory components (SPA1/COP1-dependent E3 ubiquitin ligase to CO/NF-Ys transcriptional complex) is shared (Fig. 4). Previously, the regulatory kernel has been proposed as part of a unique mechanism developed for photoperiodic flowering ${ }^{11}$. The results presented in this study have shown that the kernel's counterpart in C. reinhardtii functions in photoprotection. This raises the possibility that the root of this method of regulation could be extended back to the early evolutional history of green photosynthetic organisms. At the same time, we may need to reinterpret the significance of flowering in plants and photoprotection in green algae within the same context, because of a shared fundamental regulatory mechanism. Whether we can see the same type of regulation in other algae would be a matter of future study as our current knowledge about their photoprotective behavior as well as the related genetic information is still very limited.

\section{Methods}

Statistics. Statistical methods were not used to predetermine the sample size. The experiments were not randomized, and the investigators were not blinded to allocation during experimental procedures and data assessment.

Strains and growth conditions. Chlamydomonas reinhardtii strains $137 \mathrm{c}$, CC4286, and CLiP (CC5325 as the wild type and LMJ.RY0402.060340 as the spa1 mutant) were obtained from the Chlamydomonas Resource Center (http://www.

chlamycollection.org). All strains were grown in tris-acetate-phosphate (TAP) medium and illuminated at $50 \mu \mathrm{mol}$ photons $\mathrm{m}^{-2} \mathrm{~s}^{-1}$ at $25^{\circ} \mathrm{C}$ with rotary shaking at $150 \mathrm{rpm}$. Cells at mid-log phase $\left(\sim 4 \times 10^{6}\right.$ cells $\left./ \mathrm{mL}\right)$ were harvested and resuspended in high salt (HS) medium at $1 \times 10^{6}$ cells $/ \mathrm{mL}$ and treated with light as described in the text and figure legends. The LHCSR1-Luc717 strain, harboring a LHCSR1-luciferase fused reporter construct ${ }^{9}$, was used as a control strain for the DSR mutants. The $137 \mathrm{c}$ strain was used as a control/recipient strain for NF-YC mutants. All the DSR mutants were previously isolated through a forward genetic screen using the LHCSR1-Luc717 strain ${ }^{9}$.

Growth evaluation of the strains. The growth of WT and mutant strains used in this study was evaluated under the conditions previously described ${ }^{16}$. Briefly, cells were grown under 30 or $100 \mu \mathrm{mol}$ photons $\mathrm{m}^{-2} \mathrm{~s}^{-1}$ of continuous white fluorescent light in HS media, and cells were counted every $24 \mathrm{~h}$. To assess cell shapes, cells at the steady-state growth phase (day 6) were imaged at room temperature using a TCS SP8 confocal laser scanning microscope (Leica Microsystems, Germany) equipped with a HC PL APO CS2 $63 \times /$ NA1.40 oil objective lens. A 448-nm diode laser with $2 \%$ output was selected to obtain bright-field images. The images were produced on a PMT-based detector. All images were acquired at a $600-\mathrm{Hz}$ laser scan speed and analyzed by LASX software (Leica Microsystems).

Identification of the insertion site of the $\boldsymbol{a p h} \mathbf{7}$ cassette. The genomic regions flanking the inserted DNA tag were cloned by RESDA-PCR ${ }^{33}$. The tag DNAspecific and degenerate primers are listed in Supplementary Table 6. The PCR mixture for a first amplification contained tag-specific primer (aph7tag-F1 for downstream of the tag DNA; aph7tag-R1 for upstream of the tag DNA), degenerate primer (DegPstI, DegSacII, DegBglII, or DegMluI), rTaq DNA polymerase (TOYOBO, Japan), and genomic DNA template. The mixture was placed in a SimpliAmp Thermal Cycler (Thermo Fisher Scientific, Waltham, MA, USA), and the following program was run: $95^{\circ} \mathrm{C}$ for $8 \mathrm{~min}$; five cycles of $95^{\circ} \mathrm{C}$ for $30 \mathrm{~s}, 58^{\circ} \mathrm{C}$ for $1 \mathrm{~min}$, and $72^{\circ} \mathrm{C}$ for $3 \mathrm{~min} ; 95^{\circ} \mathrm{C}$ for $30 \mathrm{~s} ; 25^{\circ} \mathrm{C}$ for $3 \mathrm{~min} ; 72^{\circ} \mathrm{C}$ for $3 \mathrm{~min} ; 20$ cycles of $95^{\circ} \mathrm{C}$ for $30 \mathrm{~s}, 58^{\circ} \mathrm{C}$ for $1 \mathrm{~min}, 72^{\circ} \mathrm{C}$ for $3 \mathrm{~min}, 95^{\circ} \mathrm{C}$ for $30 \mathrm{~s}, 58^{\circ} \mathrm{C}$ for $1 \mathrm{~min}, 72^{\circ} \mathrm{C}$ for $3 \mathrm{~min}, 95^{\circ} \mathrm{C}$ for $30 \mathrm{~s}, 40^{\circ} \mathrm{C}$ for $1 \mathrm{~min}$, and $72^{\circ} \mathrm{C}$ for $3 \mathrm{~min} ; 72^{\circ} \mathrm{C}$ for $5 \mathrm{~min}$. The PCR mixture for a second amplification contained tag-specific primer (aph7tag-F2 or aph7tag-F3 for downstream of the tag DNA; aph7tag-R2 or aph7tag-R3 for upstream of the tag DNA), Q0 primer, KOD FX Neo DNA polymerase (TOYOBO), and the diluted first amplification product. After running standard amplification program for KOD FX Neo DNA polymerase, the second amplification products were purified using the Wizard SV Gel and PCR Clean-Up System (Promega, Madison, WI, USA) and subjected to DNA sequencing. The sequenced genomic nucleotides were then analyzed by a BLAST search in Chlamydomonas reinhardtii genome v5.5 (Phytozome, https://phytozome.jgi.doe.gov/) to identify the position of the inserted $a p h 7$ cassette in the genome.
Linkage mapping of the $\mathbf{d s r} \mathbf{4 2 8 6}$ and $\mathbf{d s r 2 8}$ mutations. Having determined that the CC4286 strain harbored a mutation that caused a defect in UV-inducible LHCSR1 expression ${ }^{9}$, we named the mutation $d s r 4286$. To establish the $d s r 4286$ mutation in the 137c background, CC4286 was crossed with LHCSR1-Luc443, another clone of LHCSR1-luciferase transformants of 137c (mt+). An F3 progeny, \#443-1A-12-4b $(\mathrm{mt}+d s r 4286)$, was crossed with the polymorphic strain S1D2 (mt-, CC2290 $\left.{ }^{34}\right)$, and tetrad progeny were dissected. Recombination frequencies between $d s r 4286$ and genetic markers were determined by detecting polymorphic PCR products in 44 progeny that were deficient in UV-inducible LHCSR1 expression ( $d s r$ phenotype). $d s r 4286$ was mapped to a region before position 1515000 on chromosome 2 of the Joint Genome Institute (JGI) version 5.5. To examine the linkage between the $d s r 4286$ and $d s r 28$ mutations, \#443-1A-12-4b was crossed with an F1 progeny of a backcross of DSR28 and LHCSR1-Luc717 (mt-). All the resulting 91 progeny were tested for LHCSR1 expression and showed the mutant phenotype, suggesting that mutation in DSR28 (dsr28) is strongly linked to $d s r 4286$.

Whole-genome sequencing of $\mathbf{d s r} 4286$ and $\boldsymbol{d s r 2 8}$. Two $d s r 4286$ mutants, the F 3 progeny of a backcross of CC4286 [\#443-1A-12-5a (dsr4286 mt+) and \#443-1A-12$5 d(d s r 4286 \mathrm{mt}-)]$, and four clones from one tetrad set of the second backcross of DSR28 [DSR28-7c-4a (dsr28 mt-), - $4 b$ (wild-type $\mathrm{mt}+$ ), $-4 c$ (wild-type $\mathrm{mt}-$ ), and $-4 d(d s r 28 \mathrm{mt}+)]$ were subjected to whole-genome sequencing analysis. The genomic DNA was prepared using a DNeasy Plant Mini Kit (Qiagen, Germany). Libraries were constructed using an Illumina TruSeq DNA PCR-Free Library Prep Kit (Illumina, San Diego, CA, USA) from $2 \mu \mathrm{g}$ of each DNA sample, according to the low-throughput protocol. Sequencing was conducted on a HiSeq

1500 sequencer (Illumina) to produce $2 \times 106$-bp paired-end reads. The sequencing data were submitted to the DDBJ Sequence Read Archive (SRA) (http://www.ddbj. nig.ac.jp/dra). All sequences were trimmed with Trimmomatic ${ }^{35}$ and aligned onto the JGI version 5.5 Chlamydomonas assembly using Bowtie2 (bowtie-bio.sourceforge.net/bowtie2/index.shtml). The resulting files were converted to Binary Sequence Alignment/Map and sorted by SAMtools (samtools.courveforge.net/). The variants were identified using SAMtools and freebayes (https:/github.com/ ekg/freebayes/).

To identify the variant responsible for the $d s r 4286$ mutation, the variants that were mapped to a region before position 1515000 on chromosome 2 were extracted from \#443-1A-12-5a (dsr4286 mt+), \#443-1A-12-5d (dsr4286 mt-), dsr28-7c-4b (wild-type $\mathrm{mt}+$ ), and $d s r 28-7 c-4 c$ (wild-type $\mathrm{mt}-$ ) strains for comparison. Twenty variants were identified that were present in \#443-1A-12-5a and \#443-1A-12-5d but not in DSR28-7c-4b (wild-type $\mathrm{mt}+$ ) or DSR28-7c-4c (wild-type mt-). These variants were confirmed on the alignment data visualized by the Integrated Genomics Viewer (IGV) (http://software.broadinstitute.org/software/igv/). The variant at position 871101 located in the first exon of Cre02.g079200 appears to be a causal mutation of $d s r 4286$ because all 31 reads contained a 5-bp deletion. To identify a variant responsible for the $d s r 28$ mutation, the alignment data of DSR28$7 c-4 a(d s r 28 \mathrm{mt}-)$ and $D S R 28-7 c-4 d(d s r 28 \mathrm{mt}+)$ were visualized by IGV, with a focus on Cre02.g079200. The $\sim 30$-bp region around position 871830 on chromosome 2, located in the third intron of Cre02.g079200, was not mapped in either strain.

To further confirm the mutation sites of $d s r 4286$ and $d s r 28$, PCR amplification followed by sequencing analysis was performed. For the $d s r 4286$ mutation, $\sim 1-\mathrm{kb}$ DNA fragments were PCR amplified using DSR28-EcoRI-F and DSR28-R2 primers, and genomic DNA was prepared from strains \#443-1A-12-5a and \#443-1A-12-5d as a template. Sequencing analysis of these fragments revealed that $5 \mathrm{nt}$ was missing from the first exon (Supplementary Fig. 2). For the $d s r 28$ mutation, primers Cre12 (2898864R) and Cre12(2898864R) were designed to anneal the insertion predicted by the visualized mapping on IGV. The $\sim 700$ - and $\sim 500$-bp fragments were PCR amplified using primer pairs DSR28-F2 and Cre12(2898864R), and Cre12 (2894770F) and DSR28-R2, respectively, and genomic DNA was prepared from DSR28-7c-4a and DSR28-7c-4d as a template. Sequencing analysis revealed that the third intron of Cre02.g079200 contained the 33-bp deletion (Supplementary Fig. 2).

NFYC gene disruption using the CRISPR-Cas9 system. CRISPR-Cas 9 targeting of NFYC exon2 was performed to generate $n f y c$ mutants as described previously ${ }^{36}$ using a guide RNA sequence (5'-TGAATACAGTTGGGTCAGGT-3') and a double-stranded homology-directed repair donor (ds-HDR). The sequences of sense (HDR-F) and antisense (HDR-R) 90-bp oligonucleotides were arranged with three PTO bonds at the $3^{\prime}$ and $5^{\prime}$ end bases. Equimolar concentrations of sense and antisense oligonucleotides were annealed in $1 \times$ DUPLEX buffer $(100 \mathrm{mM}$ potassium acetate, $30 \mathrm{mM}$ HEPES, $\mathrm{pH} 7.5$, Integrated DNA Technologies [IDT; Coralville, IA, USA]) by incubation at $95^{\circ} \mathrm{C}$ for $2 \mathrm{~min}$ to generate $10 \mu \mathrm{M}$ ds-HDR. Recombinant Streptococcus pyrogenes Cas 9 protein, tracrRNA, and crRNA were purchased from IDT. Equimolar concentrations of tracrRNA and crRNA were annealed in DUPLEX buffer by heating to $95^{\circ} \mathrm{C}$ for 2 min to generate $10 \mu \mathrm{M}$ gRNA. Subsequently, $10 \mu \mathrm{M}$ Cas 9 protein was mixed with equimolar concentrations of annealed gRNA in 1x Buffer 3.1 (New England Biolabs, Beverly, MA, USA) to a final concentration of $3 \mu \mathrm{M}$ each and incubated for $15 \mathrm{~min}$ at $37^{\circ} \mathrm{C}$ to form ribonucleoprotein (RNP). Cells were grown in a synchronized light cycle (light for $14 \mathrm{~h}$ at $25^{\circ} \mathrm{C}$ : darkness for $10 \mathrm{~h}$ at $18^{\circ} \mathrm{C}$ ) in TAP medium and then harvested and suspended in MAX Efficiency Transformation Reagent for Algae (Thermo Fisher Scientific) supplemented with $40 \mathrm{mM}$ sucrose to a density of $1 \times 10^{8}$ cells $/ \mathrm{mL}$. The 
concentrated cells were heat-shocked at $40{ }^{\circ} \mathrm{C}$ for $30 \mathrm{~min}$ and mixed using a thermoshaker $(350 \mathrm{rpm})$. Forty microliters of heat-shocked cell suspension was mixed with $5 \mu \mathrm{L}$ of $3 \mu \mathrm{M}$ RNP, $1.5 \mu \mathrm{L}$ of $10 \mu \mathrm{M}$ ds-HDR, and $0.3 \mu \mathrm{g}$ of selection marker plasmid pHyg 3 and then electroporated using the NEPA21 Super Electroporator (Nepa Gene Co., Ltd., Japan). The transformed cells were then selected under dim light at $25^{\circ} \mathrm{C}$ on TAP plates containing $10 \mu \mathrm{g} / \mathrm{mL}$ of hygromicin. The hygromycin-resistant clones were screened for insertion of the "FLAG" sequence by colony PCR using NFYC-check-F and FLAG-R primers.

Complementation of DSR mutants. For the complementation of $\mathrm{crco}-2$ (DSR15), $n f y b-1$ (DSR28), and uvr8 (DSR1) mutants, a full-length version of their genes was fused in-frame to Venus-3xFLAG under the control of the HSP70A/RBCS2 tandem promoter $^{37}$. The generated complementation vectors with the aadA gene (spectinomycin resistance marker) were introduced into the DSR15, DSR28, or DSR1 strains by electroporation using a NEPA21 Super Electroporator (Nepa Gene Co., Ltd.). All strains in the exponential phase of growth $\left(2 \times 10^{6}\right.$ cells $\left./ \mathrm{mL}\right)$ were transformed with $300 \mathrm{ng}$ of linearized vector. The transformed cells were then selected under dim light at $25^{\circ} \mathrm{C}$ on TAP plates containing $10 \mu \mathrm{g} / \mathrm{mL}$ of spectinomycin.

Genetic crossing of the mutants. To generate double mutants harboring the spa1 mutation and CrCO-Venus-3xFLAG construct, the CLiP spa1 mutant LMJ. $R Y 0402.060340$ was crossed with the $\mathrm{crco}-2 / \mathrm{CrCO}$ strain. The resulting progeny were dissected and categorized to wild-type, spal, crco-2/CrCO, spal crco-2, and spal $\mathrm{crco}-2 / \mathrm{CrCO}$ genotype strains. To generate triple mutants harboring the $\mathrm{crco}-2$ mutation, $n f y b-1$ mutation, and NFYB-Venus-3xFLAG construct, the crco-2 mutant was crossed with the $n f y b-1 / N F Y B$ strain. The resulting progeny were dissected and $c r c o-2 n f y b-1 / N F Y B$ genotype strains were isolated.

Standard mRNA quantification. Total RNA from light-treated cells was extracted using the Maxwell RSC instrument (Promega) equipped with the Maxwell RSC simplyRNA Tissue Kit (Promega). The isolated RNA was quantified using the QuantiFluor RNA System (Promega) prior to reverse transcription. Reverse transcription and semiquantitative PCR were performed using a ReverTra Ace ${ }^{\circledR}$ qPCR RT Kit with gRemover (TOYOBO) and KOD FX Neo DNA Polymerase (TOYOBO) in the SimpliAmp Thermal Cycler (Thermo Fisher Scientific). For reverse transcription, a $300 \mathrm{ng}$ of isolated RNA sample was used in a $10-\mu \mathrm{L}$ total reaction volume. Semiquantitative RT-PCR was performed using $3 \mathrm{ng}$ of cDNA thus obtained. For semiquantitative RT-PCR, the G protein $\beta$-subunit-like polypeptide $(C B L P)$ gene was chosen as the housekeeping gene during light treatment. The primers used for semiquantitative RT-PCR are listed in Supplementary Table 7.

Immunoblot analysis. Protein samples from whole cell extracts (corresponding to $\sim 2 \times 10^{6}$ cells, unless stated otherwise) were loaded onto $11 \%$ acrylamide with $7 \mathrm{M}$ urea SDS-PAGE (gel size: $15 \mathrm{~cm}$ width $\times 10 \mathrm{~cm}$ height $\times 1 \mathrm{~mm}$ thickness), and the electrophoresis was performed at a constant current $8 \mathrm{~mA}$ for $15 \mathrm{~h}$. The separated polypeptides were blotted onto nitrocellulose membranes with a tank blot system (BIO-CRAFT, Japan) at a constant voltage $40 \mathrm{~V}$ for $3 \mathrm{~h}$. The blotted membranes were treated with a blocking reagent EzChemi Block (ATTO, Japan) for $1 \mathrm{~h}$. The primary and secondary antibody treatments were done with shaking at room temperature for $1 \mathrm{~h}$. Antiserum against ATPB was obtained from Agrisera (AS05 085, rabbit polyclonal, at 1:10,000 dilution); antisera against LHCSR1 and LHCSR3 (rabbit polyclonal, at 1:10,000 dilution) were raised and affinity purified against the peptide LGLKPTDPEELK; ${ }^{9}$ antiserum against PSBS (rabbit polyclonal, at 1:5000 dilution) was provided Prof. Peter Jahns (Heinrich-Heine-University, Germany); antisera against $\mathrm{CrCO}$ (rabbit polyclonal, at 1:1000 dilution) was raised and affinity purified against the peptide AAWFVDDEKMG (Eurofins Genomics, Japan); and antiserum against FLAG fusion proteins was obtained from Sigma-Aldrich (F1804, mouse monoclonal, at 1:1000 dilution). An anti-rabbit horseradish peroxidaseconjugated antiserum (\#7074, Cell Signaling Technology, Danvers, MA, USA) or an anti-mouse horseradish peroxidase-conjugated antiserum (\#330, MBL, Japan) was used as a secondary antibody (at 1:10,000 dilution). The blots were developed using ECL detection reagent EzWestLumi Plus (ATTO), and images of the blots were obtained using a CCD imager ChemiDocTouch System (Bio-Rad Laboratories, Hercules, CA, USA). The upper band of LHCSR3 represents its phosphorylated form (LHCSR3-P) ${ }^{6}$.

High-light tolerance assay. Mutant cells suspended in HS medium were irradiated for $8 \mathrm{~h}$ with either low light (LL; white fluorescence light at $30 \mu \mathrm{mol}$ photons $\mathrm{m}^{-2} \mathrm{~s}^{-1}$ ) or high light (HL) at $300 \mu \mathrm{mol}$ photons $\mathrm{m}^{-2} \mathrm{~s}^{-1}$ including UV (using a T8 ReptiSun 10.0 UVB fluorescent bulb [Zoo Med Laboratories, CA, USA] at $30 \mu \mathrm{mol}$ photons $\mathrm{m}^{-2} \mathrm{~s}^{-1}$ ) at $25^{\circ} \mathrm{C}$. Total cell protein extracts were obtained from the cell cultures after $1,2,4,6$, and $8 \mathrm{~h}$ of light treatment. Chlorophyll concentrations and cell numbers were calculated by the Porra calculation ${ }^{38}$ and TC20 automated cell counter (Bio-Rad Laboratories). The chlorophyll levels were then normalized to cell numbers (pg of chlorophyll per cell). LL- and HL-treated strains were adjusted to $1 \times 10^{7}$ cells $/ \mathrm{mL}$, transferred into multiwell plates, and photographed, as indicated in the figure captions (Fig. 1 and Supplementary Fig. 6).
Chlorophyll fluorescence-based photosynthetic analysis. The cells were transferred into a 48-muliwell plate and treated with far-red light $(<5 \mu \mathrm{mol}$ photons $\mathrm{m}^{-2} \mathrm{~s}^{-1}$ ) for $30 \mathrm{~min}$. To evaluate the maximum quantum yield of photosystem II (Fv/Fm), minimal and maximal fluorescence yields (Fo; Fm) were measured under dark conditions using a FluoCam MF800 (Photon System Instruments, Czech Republic). Fv/Fm was calculated using the equation $\mathrm{Fv} / \mathrm{Fm}=(\mathrm{Fm}-\mathrm{Fo}) / \mathrm{Fm}$. To evaluate the photoprotection capability $(\mathrm{qE})$, maximal fluorescence yield under light $\left(\mathrm{Fm}^{\prime}\right)$ was also measured after actinic irradiation at $750 \mu \mathrm{mol}$ photons $\mathrm{m}^{-2} \mathrm{~s}^{-1}$ for $30 \mathrm{~s}$. $\mathrm{qE}$ was estimated using the equation $\mathrm{qE}=\left(\mathrm{Fm}-\mathrm{Fm}^{\prime}\right) / \mathrm{Fm}^{\prime}$.

Yeast two-hybrid assay. To construct the GAL4-activation domain (AD)- or GAL4-binding domain (BD)-fused CrCO, NF-YB, NF-YC, SPA1, or COP1 expression plasmids for the yeast two-hybrid assay, the corresponding full-length coding sequences were amplified from cDNA obtained from Chlamydomonas strain 137c by PCR (primers CrCO-EcoRI-F/CrCO-SalI-R for CrCO, NF-YB-EcoRI-F/NF-YBSalI-R, NF-YC-EcoRI-F/NF-YC-SalI-R, COP1-F-Y2H/COP1-R-Y2H for COP1 and SPA1-F-Y2H/SPA1-R-Y2H for SPA1) using KOD FX Neo DNA polymerase (TOYOBO) on a SimpliAmp Thermal Cycler (Thermo Fisher Scientific). The amplicons $(\sim 1.25 \mathrm{~kb}$ for $C r C O, \sim 0.68 \mathrm{~kb}$ for NFYB, $\sim 0.91 \mathrm{~kb}$ for NFYC, $\sim 4.37 \mathrm{~kb}$ for $C O P 1$ and $\sim 4.17 \mathrm{~kb}$ for SPA1) were cloned into the pGAD-C2 or pGBD-C2 vectors ${ }^{39} . \mathrm{AD}$ and $\mathrm{BD}$ fusion plasmids were introduced into Saccharomyces cerevisiae reporter strain pJ69-4A (MATa trp1 leu2 ura3 his3 gal4D gal80D LYS2::GAL1-HIS3 GAL2-ADE2 met2::GAL7-lacZ) ${ }^{39}$. The transformants were then selected on synthetic dextrose (SD) plates lacking tryptophan (Trp) and leucine (Leu) and used for the yeast two-hybrid assay. An empty AD or BD vector served as a negative control. The yeast cells were suspended in liquid medium at a concentration of $2 \times 10^{7}$ cells/ $\mathrm{mL}$, and $5-\mu \mathrm{L}$ droplets of 5 -fold serial dilutions were spotted onto SD-Leu-Trp (+His) and SD-Leu-Trp-His (-His) plates. The plates were incubated at $30^{\circ} \mathrm{C}$ for $40 \mathrm{~h}$. The primers used for PCR analyses are listed in Supplementary Table 8.

Immunoprecipitation and mass spectroscopy analysis. Cells treated with each experimental light condition (HL for $n f y b-1 / N F Y B$ and UV for $u v r 8 / U V R 8$ and crco-2/CrCO) for $1 \mathrm{~h}$ were harvested and resuspended in standard PBS buffer. The cells were disrupted by a previously reported airbrush method ${ }^{40}$ using a $0.2-\mathrm{mm}$ caliber airbrush (PS270, GSI Creos Corporation, Japan) three times at $7.5 \mathrm{kgf} \mathrm{cm}^{-2}$ and then solubilized with Triton X-100 (Sigma-Aldrich, St. Louis, MO, USA) for $5 \mathrm{~min}$ to a final concentration of $0.5 \%$ in PBS buffer. Unsolubilized cell debris was removed by centrifugation at $20,000 \times \mathrm{g}$ at $4^{\circ} \mathrm{C}$ for $5 \mathrm{~min}$. The Venus-3xFLAGfused proteins were immunoprecipitated using $100 \mu \mathrm{L}$ of SURE-beads (Bio-Rad Laboratories) conjugated with $5 \mu \mathrm{g}$ of FLAG (M2) mouse monoclonal antibody (Sigma-Aldrich) at $4{ }^{\circ} \mathrm{C}$ for $1 \mathrm{~h}$. The immunoprecipitates were then eluted using $3 x$ FLAG peptide (ProteinArk, Sheffield, UK) at $500 \mathrm{ng} / \mu \mathrm{L}$ with shaking at room temperature for $15 \mathrm{~min}$, and the eluted polypeptides were separated on a $12.5 \%$ PAGEL-HR gel (ATTO) at a constant current $10 \mathrm{~mA}$ for $90 \mathrm{~min}$ and in-gel trypsin digested according to Shevchenko et al. ${ }^{41}$. The in-gel-digested samples were subjected to LC-MS/MS spectroscopy analysis using an Orbitrap Elite mass spectrometer (Thermo Fisher Scientific). Tandem mass spectrometry results were submitted for protein database searching using Proteome Discoverer software (Thermo Fisher Scientific) and Mascot v.2.5.1 (Matrix Science, London, UK) against a database generated from Chlamydomonas reinhardtii genome v5.5 (Phytozome, https://phytozome.jgi.doe.gov/).

Chromatin immunoprecipitation PCR assay. Chromatin immunoprecipitation (ChIP) experiments were performed as reported previously ${ }^{42}$. Cells treated for $1 \mathrm{~h}$ in the high-light tolerance assay were harvested by centrifugation at $2000 \times g$ at $4{ }^{\circ} \mathrm{C}$ for $2 \mathrm{~min}$ and resuspended in $\mathrm{KH}$ buffer $(20 \mathrm{mM}$ HEPES-KOH, pH7.6, and $80 \mathrm{mM}$ $\mathrm{KCl}$ ) containing $0.35 \%$ formaldehyde (Wako Chemical, Japan). After incubation for $10 \mathrm{~min}$ at room temperature, the cross-linking reaction was terminated with $1 \mathrm{M}$ glycine for $5 \mathrm{~min}$. The cross-linked cells were sonicated using the BIORUPTOR ${ }^{\circledR}$ II (Cosmo Bio, Japan) for $10 \mathrm{~s}(\times 30)$ with pauses of $20 \mathrm{~s}$ between each sonication cycle and then subjected to ChIP. The target DNA-protein complexes were immunoprecipitated using $100 \mu \mathrm{L}$ of SURE-beads (Bio-Rad Laboratories) conjugated with $5 \mu \mathrm{g}$ of FLAG (M2) mouse monoclonal antibody (Sigma-Aldrich) at $4^{\circ} \mathrm{C}$ for $1 \mathrm{~h}$. After an overnight cross-link reversion between DNA and protein at $65^{\circ} \mathrm{C}$, the DNA was extracted and purified using the phenol/chloroform/isoamylalcohol purification method. The purified DNA was then subjected to semiquantitative PCR using KOD FX Neo DNA polymerase (TOYOBO) on the SimpliAmp Thermal Cycler (Thermo Fisher Scientific). Real-time qPCR assays were performed using the KOD SYBR ${ }^{\circledR}$ qPCR Mix (TOYOBO) on the Light Cycler 96 system (Roche Diagnostics, Germany). The primers used for PCR analyses are listed in Supplementary Table 7.

Live-cell imaging of Venus-3xFLAG fusion proteins. Chlamydomonas reinhardtii cells expressing Venus-3xFLAG were mixed with low-gelling temperature agarose (A6560, Sigma-Aldrich) and transferred into a collagen-coated grass bottom dish (D11134H, Matsunami Glass, Japan). The agarose-embedded cells were imaged at room temperature on a TCS SP8 confocal laser scanning microscope (Leica Microsystems) equipped with a HC PL APO CS2 63×/NA1.40 oil objective lens. A $514-\mathrm{nm}$ diode laser with $2 \%$ output was used to excite both Venus and chlorophyll, 
and emission from 516 to $565 \mathrm{~nm}$ and $680-700 \mathrm{~nm}$ was collected for Venus fluorescence and chlorophyll autofluorescence, respectively, on a HyD SMD hybrid detector (Leica Microsystems). Bright-field images were produced using a PMTbased detector. All images were acquired with four-line accumulation at a $600-\mathrm{Hz}$ laser scan speed in photon counting mode and analyzed using LASX software (Leica Microsystems). Cells expressing UVR8-Venus-3xFLAG in the DSR1 (uvr8) background were imaged after $0,5,10,15,20$, and $30 \mathrm{~min}$ of UV illumination.

Immunocytochemistry of Venus-3xFLAG fusion proteins. Cells were harvested by centrifugation at $2000 \times g$ at $4^{\circ} \mathrm{C}$ for $2 \mathrm{~min}$. and fixed by suspension in PBS containing $1 \%$ paraformaldehyde at $4{ }^{\circ} \mathrm{C}$. The fixed cells were seeded on poly-Llysine-coated coverslips (Asahi Techno Glass Corp., Japan) and were treated with methanol twice each for $10 \mathrm{~min}$ at $-20{ }^{\circ} \mathrm{C}$ to permeabilize the membrane and remove chlorophyll. After rehydration with PBS, the coverslips were treated with a blocking buffer ( $5 \%$ bovine serum albumin, $1 \%$ cold water fish skin gelatin, and $10 \%$ goat serum in PBS), a primary antibody (mouse monoclonal anti-FLAG (M2) antibody; Sigma-Aldrich) at $4{ }^{\circ} \mathrm{C}$ overnight, and a secondary antibody [AlexaFluor546-conjugated $\mathrm{F}(\mathrm{ab}$ ') 2 fragment of goat anti-mouse IgG; Thermo Fisher Scientific] at room temperature for $2 \mathrm{~h}$. Coverslips were then mounted onto the slides with ProLong Diamond Antifade Mountant with DAPI (Thermo Fisher Scientific). Fluorescence was observed with a confocal laser scanning fluorescence microscope (FV10i-DOC, Olympus, Japan).

Proteasome inhibitor assay. The proteasome inhibitor MG132 (Wako Chemical) dissolved in dimethylsulfoxide (DMSO) was added to the culture before starting the light treatment. The final concentration of DMSO was adjusted to $0.5 \%$ with a final concentration of MG132 of $0,10,25,50,100$, and $200 \mu \mathrm{M}$, as indicated in Supplementary Fig. 13. The cells were cultured under LL for $2 \mathrm{~h}$ after MG132 was added.

Circadian mRNA rhythm assay of CrCO. Asynchronous cultures in HS media $\left(2 \times 10^{6}\right.$ cells $\left./ \mathrm{mL}\right)$ were kept in darkness for $12 \mathrm{~h}$ at $17^{\circ} \mathrm{C}$ to synchronize the circadian clock and then exposed to continuous light $\left(10 \mu \mathrm{mol}\right.$ photons $\left.\mathrm{m}^{-2} \mathrm{~s}^{-1}\right)$. Cells were harvested every $4 \mathrm{~h}$ from 24 to $72 \mathrm{~h}$. mRNA quantification by RT-qPCR was performed as described previously ${ }^{14}$. Total RNA was extracted using TRIzol ${ }^{\circledR}$ reagent (Thermo Fisher Scientific). ReverTra Ace ${ }^{\circledast}$ qPCR RT Master Mix (TOYOBO) was used for the reverse transcription procedures. qPCR was then performed using KOD FX Neo DNA polymerase (TOYOBO) supplemented with SYBR $^{\circledR}$ Green I Nucleic Acid Gel Stain (TaKaRa, Japan) and ROX dye (Thermo Fisher Scientific) to final concentrations of $0.05 \times(200,000$-fold dilution $)$ and $0.5 \mu \mathrm{M}$, respectively. Samples were quantified using StepOnePlus ${ }^{\mathrm{Tm}}$ (Thermo Fisher Scientific) by the relative standard curve method. Quantification standards for each target cDNA were obtained using band-purified PCR products as templates. The constitutively expressed RCK1 gene was used as an endogenous control for normalization. The primers used for PCR are listed in Supplementary Table 7.

Phylogenetic analysis of NF-Y proteins. The whole amino acid sequences of the NF-Y proteins from C. reinhardtii and A. thaliana were aligned using MAFFT v7.407 (https://mafft.cbrc.jp/alignment/software/). The phylogenetic tree was generated with RAxML v8.2.12 (https://cme.h-its.org/exelixis/web/software/raxml/ index.html) with a 1000-bootstrap test. The generated phylogenetic tree was constructed and visualized with MEGA7 (https://www.megasoftware.net/).

Reporting summary. Further information on research design is available in the Nature Research Reporting Summary linked to this article.

\section{Data availability}

The sequencing data have been deposited in [DDBJ Sequence Read Archive (SRA) with the accession codes DRX140139 (\#443-1A-12-5a), DRX140140 (\#443-1A-12-5d), DRX140141 (DSR28-7c-4a), DRX140142 (DSR28-7c-4b), DRX140143 (DSR28-7c-4c), and DRX140144 (DSR28-7c-4d)]. The proteomic data that support the findings of this study are available in [Japan ProteOme STandard Repository (jPOSTrepo) with the accession codes JPST000646 (Supplementary Table 1), JPST000647 (Supplementary Table 2), JPST000648 (Supplementary Table 3), JPST000649 (Supplementary Table 4), and JPST000650 (Supplementary Table 5). The source data underlying Figs. 1b-e, 2c, 3b, $3 \mathrm{~d}$ and Supplementary Figs. 2b, 3, 4a, 6c, 6d, 9, 10, 12, 13, and 14 are provided as a Source Data file.

Received: 11 January 2019 Accepted: 16 August 2019

Published online: 10 September 2019

\section{References}

1. Horton, P., Ruban, A. V. \& Walters, R. G. Regulation of light harvesting in green plants. Annu. Rev. Plant Physiol. Plant Mol. Biol. 47, 655-684 (1996).
2. Niyogi, K. K. \& Truong, T. B. Evolution of flexible non-photochemical quenching mechanisms that regulate light harvesting in oxygenic photosynthesis. Curr. Opin. Plant Biol. 16, 307-314 (2013).

3. Peers, G. et al. An ancient light-harvesting protein is critical for the regulation of algal photosynthesis. Nature 462, 518-521 (2009).

4. Li, X. P. et al. A pigment-binding protein essential for regulation of photosynthetic light harvesting. Nature 403, 391-395 (2000).

5. Allorent, G. \& Petroutsos, D. Photoreceptor-dependent regulation of photoprotection. Curr. Opin. Plant Biol. 37, 102-108 (2017).

6. Petroutsos, D. et al. A blue-light photoreceptor mediates the feedback regulation of photosynthesis. Nature 537, 563-566 (2016).

7. Allorent, G. et al. UV-B photoreceptor-mediated protection of the photosynthetic machinery in Chlamydomonas reinhardtii. Proc. Natl Acad. Sci. USA 113, 14864-14869 (2016).

8. Aihara, Y., Fujimura-Kamada, K., Yamasaki, T. \& Minagawa, J. Algal photoprotection is regulated by the E3 ligase CUL4-DDB1DET1. Nat. Plants 5, 34-40 (2019).

9. Tokutsu, R., Fujimura-Kamada, K., Yamasaki, T., Matsuo, T. \& Minagawa, J. Isolation of photoprotective signal transduction mutants by systematic bioluminescence screening in Chlamydomonas reinhardtii. Sci. Rep. 9, 2820 (2019).

10. Suarez-Lopez, P. et al. CONSTANS mediates between the circadian clock and the control of flowering in Arabidopsis. Nature 410, 1116-1120 (2001).

11. Song, Y. H., Shim, J. S., Kinmonth-Schultz, H. A. \& Imaizumi, T. Photoperiodic flowering: time measurement mechanisms in leaves. Annu. Rev. Plant Biol. 66, 441-464 (2015).

12. Nardini, M. et al. Sequence-specific transcription factor NF-Y displays histone-like DNA binding and H2B-like ubiquitination. Cell 152, 132-143 (2013).

13. Zhao, $\mathrm{H}$. et al. The Arabidopsis thaliana nuclear factor $\mathrm{Y}$ transcription factors. Front. Plant Sci. 7, 2045 (2017).

14. Li, X. Y. et al. Evolutionary varidation of the CCAAT-binding transctirption factor NF-Y. Nucleic Acids Res. 20, 1087-1091 (1992).

15. Wenkel, S. et al. CONSTANS and the CCAAT box binding complex share a functionally important domain and interact to regulate flowering of Arabidopsis. Plant Cell 18, 2971-2984 (2006).

16. Serrano, G. et al. Chlamydomonas CONSTANS and the evolution of plant photoperiodic signaling. Curr. Biol. 19, 359-368 (2009).

17. Thiriet-Rupert, S. et al. Transcription factors in microalgae: genome-wide prediction and comparative analysis. BMC Genom. 17, 282 (2016).

18. Valverde, F. CONSTANS and the evolutionary origin of photoperiodic timing of flowering. J. Exp. Bot. 62, 2453-2463 (2011).

19. Kromdijk, J. et al. Improving photosynthesis and crop productivity by accelerating recovery from photoprotection. Science 354, 857-861 (2016).

20. Kumimoto, R. W., Zhang, Y., Siefers, N. \& Holt, B. F. NF-YC3, NF-YC4 and NF-YC9 are required for CONSTANS-mediated, photoperiod-dependent flowering in Arabidopsis thaliana. Plant J. 63, 379-391 (2010).

21. Ben-Naim, O. et al. The CCAAT binding factor can mediate interactions between CONSTANS-like proteins and DNA. Plant J. 46, 462-476 (2006).

22. Gnesutta, N. et al. CONSTANS imparts DNA sequence specificity to the hHistone fold NF-YB/NF-YC dimer. Plant Cell 29, 1516-1532 (2017).

23. Myers, Z. A. \& Holt, B. F. NUCLEAR FACTOR-Y: still complex after all these years? Curr. Opin. Plant Biol. 45, 96-102 (2018)

24. Valverde, F. et al. Photoreceptor regulation of CONSTANS protein in photoperiodic flowering. Science 303, 1003-1006 (2004).

25. Lau, O. S. \& Deng, X. W. The photomorphogenic repressors COP1 and DET1: 20 years later. Trends Plant Sci. 17, 584-593 (2012).

26. Jang, S. et al. Arabidopsis COP1 shapes the temporal pattern of CO accumulation conferring a photoperiodic flowering response. EMBO J. 27, 1277-1288 (2008)

27. Liu, L. J. et al. COP1-mediated ubiquitination of CONSTANS is implicated in cryptochrome regulation of flowering in Arabidopsis. Plant Cell 20, 292-306 (2008).

28. Zuo, Z. C., Liu, H. T., Liu, B., Liu, X. M. \& Lin, C. T. Blue light-dependent interaction of CRY2 with SPA1 regulates COP1 activity and floral initiation in Arabidopsis. Curr. Biol. 21, 841-847 (2011).

29. Rizzini, L. et al. Perception of UV-B by the Arabidopsis UVR8 protein. Science 332, 103-106 (2011).

30. Huang, X. et al. Conversion from CUL4-based COP1-SPA E3 apparatus to UVR8-COP1-SPA complexes underlies a distinct biochemical function of COP1 under UV-B. Proc. Natl Acad. Sci. USA 110, 16669-16674 (2013).

31. Gagne, G. \& Guertin, M. The early genetic response to light in the green unicellular alga Chlamydomonas eugametos grown under light/dark cycles involves genes that represent direct responses to light and photosynthesis. Plant Mol. Biol. 18, 429-445 (1992).

32. Savard, F., Richard, C. \& Guertin, M. The Chlamydomonas reinhardtii LI818 gene represents a distant relative of the cabI/II genes that is regulated during the cell cycle and in response to illumination. Plant Mol. Biol. 32, 461-473 (1996). 
33. Gonzalez-Ballester, D., de Montaigu, A., Galvan, A. \& Fernandez, E. Restriction enzyme site-directed amplification PCR: A tool to identify regions flanking a marker DNA. Anal. Biochem. 340, 330-335 (2005).

34. Gross, C. H., Ranum, L. P. W. \& Lefebvre, P. A. Extensive restriction fragment length polymorphisms in a new isolate ofChlamydomonas reinhardtii. Curr. Genet. 13, 503-508 (1988).

35. Bolger, A. M., Lohse, M. \& Usadel, B. Trimmomatic: a flexible trimmer for Illumina sequence data. Bioinformatics 30, 2114-2120 (2014).

36. Greiner, A. et al. Targeting of photoreceptor genes inChlamydomonas reinhardtiivia zinc-finger nucleases and CRISPR/Cas9. Plant Cell 29, 2498-2518 (2017).

37. Onishi, M. \& Pringle, J. R. Robust transgene expression from bicistronic mRNA in the green algaChlamydomonas reinhardtii. G3 (Bethesda) 6 , 4115-4125 (2016).

38. Porra, R. J., Thompson, W. A. \& Kriedemann, P. E. Determination of accurate extinction coefficients and simultaneous equations for assaying chlorophyllsaandbextracted with four different solvents: verification of the concentration of chlorophyll standards by atomic absorption spectroscopy. Biochim. Biophys. Acta 975, 384-394 (1989).

39. James, P., Halladay, J. \& Craig, E. A. Genomic libraries and a host strain designed for highly efficient two-hybrid selection in yeast. Genetics 144, 1425-1436 (1996).

40. Nishimura, Y. et al. An $m t^{+}$gamete-specific nuclease that targets $m t^{-}$ chloroplasts during sexual reproduction in C. reinhardtii. Genes Dev. 16, 1116-1128 (2002).

41. Shevchenko, A., Tomas, H., Havlis, J., Olsen, J. V. \& Mann, M. In-gel digestion for mass spectrometric characterization of proteins and proteomes. Nat. Protoc. 1, 2856-2860 (2006).

42. Strenkert, D., Schmollinger, S. \& Schroda, M. Protocol: methodology for chromatin immunoprecipitation (ChIP) in Chlamydomonas reinhardtii. Plant Methods 7, https://doi.org/10.1186/1746-4811-7-35 (2011).

\section{Acknowledgements}

We are grateful to Drs. Shuji Shigenobu, Shinichiro Maruyama, and Katsushi Yamaguchi for their help with whole-genome sequence analysis of the NF-YB mutants. We also appreciate Mrs. Yuko Mori and Ms. Yumiko Makino for their technical support with LCMS/MS analysis. We also thank Mrs. Tamaka Kadowaki and Mrs. Harumi Yonezawa for providing technical assistance with the genetic crossing and culturing of the algae. We thank Dr. Masafumi Hirono for the technical advice on the genetic mapping and linkage analysis. We thank Ms. Ayumi Kinoshita for her help with the rhythm assay. The PSBS antibody was kindly provided by Dr. Peter Jahns. Drs. Kyoko Hamada, Kensuke Kataoka, and Junichi Nakayama are thanked for the fruitful discussions about the ChIP assay. We thank Dr. Krishna Niyogi for sharing results prior to publication. We also thank Drs. Kenji Takizawa, Yosuke Tamada, Tomonao Matsushita, and Ryoichi Sato for the fruitful discussion and critical reading of the manuscript. This work was supported by Functional Genomics Facility, NIBB Core Research Facilities, NIBB Model Plant Research Facility,
NINS Program for Cross-disciplinary Study (grant number 01311701 to R.T.), and the JSPS Grant-in-Aid for Young Scientists (A) (grant number JP15H05599 to R.T.) and for Scientific Research on Innovative Areas (grant number JP16H06553 to J.M. and R.T.)

\section{Author contributions}

R.T. and J.M. conceived the research. R.T. designed the experiments. R.T., K.F-K. and T.Y. screened and identified the mutation sites of the mutants. R.T. performed the transcriptional, ChIP-PCR, chlorophyll fluorescence, pigment bleaching, live-cell imaging, biochemical, and phylogenetical analyses. K.F-K. performed the algal linkage mapping, whole-genome sequencing, yeast two-hybrid analyses, and mutant generation. T.M. performed the immunocytochemistry and analyzed the circadian clock response of the green alga. R.T. wrote the initial draft of the paper. J.M. supervised the entire work and revised the paper. All authors contributed to the writing and revision of the paper and approved the final version of the paper.

\section{Additional information}

Supplementary Information accompanies this paper at https://doi.org/10.1038/s41467 019-11989-x.

Competing interests: The authors declare no competing interests.

Reprints and permission information is available online at http://npg.nature.com/ reprintsandpermissions/

Peer review information: Nature Communications thanks Swadhin Swain, Federico Valverde and other, anonymous, reviewer(s) for their contribution to the peer review of this work. Peer reviewer reports are available.

Publisher's note: Springer Nature remains neutral with regard to jurisdictional claims in published maps and institutional affiliations.
Open Access This article is licensed under a Creative Commons Attribution 4.0 International License, which permits use, sharing, adaptation, distribution and reproduction in any medium or format, as long as you give appropriate credit to the original author(s) and the source, provide a link to the Creative Commons license, and indicate if changes were made. The images or other third party material in this article are included in the article's Creative Commons license, unless indicated otherwise in a credit line to the material. If material is not included in the article's Creative Commons license and your intended use is not permitted by statutory regulation or exceeds the permitted use, you will need to obtain permission directly from the copyright holder. To view a copy of this license, visit http://creativecommons.org/ licenses/by/4.0/

(C) The Author(s) 2019 